\title{
Neutrino scattering in strong magnetic fields*
}

\author{
Kaushik Bhattacharya and Palash B. Pal \\ Saha Institute of Nuclear Physics, Calcutta 700064, India
}

\section{Motivation}

Neutrino interactions in strong magnetic fields have gained a lot of attention because of the problem of neutrino emission from a proto-neutron star. It has been argued that a small asymmetry in the neutrino emission can explain the high peculiar velocities of pulsars. Based on earlier work [1] on neutrino dispersion relations in an external magnetic field, it has been shown[2] that neutrino oscillations can probably account for the pulsar kicks. On the other hand, there is a parallel stream of argument with the idea that neutrino opacities may change appreciably in magnetic fields. Asymmetric opacities can presumably account for the kicks. The opacity has recently been calculated by Roulet [3], assuming the matrix element for the interactions remain unchanged in a magnetic field. He found that the opacities do change appreciably compared to the no-field case. However, he did not find any asymmetry vis-a-vis the direction of the magnetic field. The aim of the present work is to redo these calculations from first principles and show that the relevant cross-section is indeed asymmetric.

\section{Solutions of the Dirac equation in a uniform magnetic field}

We take the magnetic field $B$ in the $z$-direction, and choose

$$
A_{0}=A_{y}=A_{z}=0, \quad A_{x}=-B y .
$$

The Dirac equation in this field can be exactly solved. The energy eigenvalues are given by

$$
E_{n}^{2}=m^{2}+p_{z}^{2}+2 n e B
$$

*Plenary talk given by Palash B. Pal at the COSMO-99 conference held at the Abdus Salam ICTP, Trieste, Italy, during 29/9/1999 to 2/10/1999 
where $n$ is a non-negative integer signifying the Landau level. All $n>0$ levels are doubly degenerate, whereas the $n=0$ level is non-degenerate. The eigenstates are of the form

$$
e^{-i p \cdot X_{\grave{y}}} U_{s}\left(y, n, \boldsymbol{p}_{\grave{y}}\right),
$$

where the notation $p \cdot X_{\dot{y}}$ stands for the dot product, setting the $y$-components equal to zero. $U_{s}$ is the spinor, given by

$$
U_{+}=\left(\begin{array}{c}
I_{n}(\xi) \\
0 \\
\frac{p_{z}}{E_{n}+m} I_{n}(\xi) \\
\frac{M_{n}}{E_{n}+m} I_{n-1}(\xi)
\end{array}\right), \quad U_{-}=\left(\begin{array}{c}
0 \\
I_{n-1}(\xi) \\
\frac{M_{n}}{E_{n}+m} I_{n}(\xi) \\
-\frac{p_{z}}{E_{n}+m} I_{n-1}(\xi)
\end{array}\right)
$$

We have used the shorthand $M_{n}=\sqrt{2 n e B}$, and a dimensionless variable

$$
\xi=\sqrt{e B}\left(y+\frac{p_{x}}{e B}\right) .
$$

The function $I_{n}(\xi)$ appearing in Eq. (何) is given by

$$
I_{n}(\xi)=N_{n} e^{-\xi^{2} / 2} H_{n}(\xi),
$$

where $H_{n}$ are Hermite polynomials, and $N_{n}$ is a normalization which can be chosen arbitrarily. We choose

$$
N_{n}=\left(\frac{\sqrt{e B}}{n ! 2^{n} \sqrt{\pi}}\right)^{1 / 2} .
$$

For the sake of consistency, we will define $I_{-1}=0$ so that the solution $U_{-}$ vanishes for the zeroth Landau level. From the spinor solutions, the spin sum can be calculated:

$$
\begin{aligned}
P_{U}\left(y, y_{\star}, n, \boldsymbol{p}_{\star}\right) \equiv \sum_{s} U_{s}\left(y, n, \boldsymbol{p}_{\star}\right) \bar{U}_{s}\left(y_{\star}, n, \boldsymbol{p}_{\star}\right) \\
\quad=\frac{1}{2\left(E_{n}+m\right)}\left[\left\{m\left(1+\sigma_{z}\right)+\not_{\|}-\widetilde{\not}_{\|} \gamma_{5}\right\} I_{n}(\xi) I_{n}\left(\xi_{\star}\right)\right. \\
\quad+\left\{m\left(1-\sigma_{z}\right)+\not_{\|}+\widetilde{p}_{\|} \gamma_{5}\right\} I_{n-1}(\xi) I_{n-1}\left(\xi_{\star}\right) \\
\left.\quad+M_{n}\left\{\left(\gamma_{1}+i \gamma_{2}\right) I_{n}(\xi) I_{n-1}\left(\xi_{\star}\right)+\left(\gamma_{1}-i \gamma_{2}\right) I_{n-1}(\xi) I_{n}\left(\xi_{\star}\right)\right\}\right],
\end{aligned}
$$

using the notations $\not p_{\|}=\gamma_{\mu} p_{\|}^{\mu}, \widetilde{p}_{\|}=\gamma_{\mu} \widetilde{p}_{\|}^{\mu}$, where

$$
p_{\|}^{\mu}=\left(p_{0}, 0,0, p_{z}\right), \quad \widetilde{p}_{\|}^{\mu}=\left(p_{z}, 0,0, p_{0}\right) .
$$




\section{The fermion field operator}

The fermion field operator can be written as

$$
\begin{aligned}
& \psi(X)=\sum_{s= \pm} \sum_{n=0}^{\infty} \int \frac{d p_{x} d p_{z}}{2 \pi} \sqrt{\frac{E_{n}+m}{2 E_{n}}}
\end{aligned}
$$

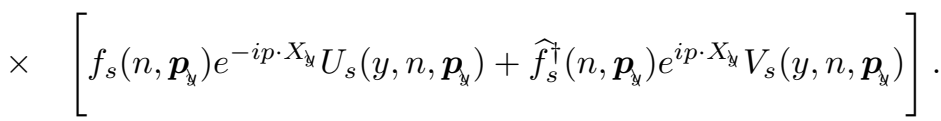

where $f_{s}\left(n, \boldsymbol{p}_{x}\right)$ is annihilation operator for the fermion, and $\widehat{f}_{s}^{\dagger}\left(n, \boldsymbol{p}_{x}\right)$ is the creation operator for the antifermion in the $n$-th Landau level with given values of $p_{x}$ and $p_{z}$. The creation and annihilation operator satisfy the anticommutation relations

$$
\left[f_{s}\left(n, \boldsymbol{p}_{\boldsymbol{x}^{\prime}}\right), f_{s^{\prime}}^{\dagger}\left(n^{\prime}, \boldsymbol{p}_{x}^{\prime}\right)\right]_{+}=\delta_{s s^{\prime}} \delta_{n n^{\prime}} \delta\left(p_{x}-p_{x}^{\prime}\right) \delta\left(p_{z}-p_{z}^{\prime}\right),
$$

etc. The prefactor appearing in Eq. (10) is determined from the fact that the field operator should satisfy the anticommutation rule

$$
\left[\psi(X), \psi^{\dagger}\left(X_{\star}\right)\right]_{+}=\delta^{3}\left(\boldsymbol{X}-\boldsymbol{X}_{\star}\right)
$$

for $X^{0}=X_{\star}^{0}$. The one-fermion states are defined by

$$
\left|n, \boldsymbol{p}_{x}\right\rangle=\frac{2 \pi}{\sqrt{L_{x} L_{z}}} f^{\dagger}\left(n, \boldsymbol{p}_{x}\right)|0\rangle,
$$

the normalization constant chosen so that these states are orthonormal in a box with sides $L_{x}, L_{y}$ and $L_{z}$. Then

$$
\left\langle n, \boldsymbol{p}_{x \mid}\right| \bar{\psi}_{U}(X)=\sqrt{\frac{E_{n}+m}{2 E_{n} L_{x} L_{z}}} e^{i p \cdot X_{k}} \bar{U}_{s}\left(y, n, \boldsymbol{p}_{x}\right)\langle 0|,
$$

where $\psi_{U}$ denotes the term in Eq. (10) that contains the $U$-spinors.

\section{Inverse beta-decay}

We now apply the above formalism for the inverse beta-decay process

$$
\nu_{e}(\boldsymbol{k})+n(\boldsymbol{P}) \rightarrow p\left(\boldsymbol{P}^{\prime}\right)+e\left(\boldsymbol{p}_{x}^{\prime}, n^{\prime}\right) .
$$

Assume $e B \ll m_{p}^{2}$, so that the magnetic field effects on the proton and neutron spinors can be ignored.

The interaction Lagrangian is

$$
\mathscr{L}_{\text {int }}=\sqrt{2} G_{\beta}\left[\bar{\psi}_{(e)} \gamma^{\mu} L \psi_{\left(\nu_{e}\right)}\right]\left[\bar{\psi}_{(p)} \gamma_{\mu}\left(g_{V}+g_{A} \gamma_{5}\right) \psi_{(n)}\right],
$$


where $G_{\beta}=G_{F} \cos \theta_{c}, g_{V}=1$ and $g_{A}=1.26$. For the $S$-matrix element, this gives

$$
\begin{aligned}
S_{f i}= & \sqrt{2} G_{\beta} \int d^{4} X\left\langle e\left(\boldsymbol{p}_{x}^{\prime}, n^{\prime}\right)\left|\bar{\psi}_{(e)} \gamma^{\mu} L \psi_{\left(\nu_{e}\right)}\right| \nu_{e}(\boldsymbol{k})\right\rangle \\
& \times\left\langle p\left(P^{\prime}\right)\left|\bar{\psi}_{(p)} \gamma_{\mu}\left(g_{V}+g_{A} \gamma_{5}\right) \psi_{(n)}\right| n(P)\right\rangle .
\end{aligned}
$$

The hadronic part of the matrix element is obtained with usual rules, which gives

$$
\frac{e^{i\left(P^{\prime}-P\right) \cdot X}}{\sqrt{2 P_{0} V} \sqrt{2 P_{0}^{\prime} V}}\left[\bar{u}_{(p)}\left(\boldsymbol{P}^{\prime}\right) \gamma_{\mu}\left(g_{V}+g_{A} \gamma_{5}\right) u_{(n)}(\boldsymbol{P})\right],
$$

For the leptonic part, we need magnetic spinors for the electron. Using Eq. (14), we obtain this matrix element to be

$$
\frac{e^{-i k \cdot X+i p^{\prime} \cdot X_{\Downarrow}}}{\sqrt{2 \omega V}} \sqrt{\frac{E_{n^{\prime}}+m}{2 E_{n^{\prime}} L_{x} L_{z}}}\left[\bar{U}_{(e)}\left(y, n^{\prime}, \boldsymbol{p}_{y}^{\prime}\right) \gamma^{\mu} L u_{\left(\nu_{e}\right)}(\boldsymbol{k})\right] .
$$

Putting these back into Eq. (17) and performing the integrations over all coordinates except $y$, we obtain

$$
S_{f i}=(2 \pi)^{3} \delta_{x}^{3}\left(P+k-P^{\prime}-p^{\prime}\right)\left[\frac{E_{n^{\prime}}+m}{2 \omega V 2 P_{0} V 2 P_{0}^{\prime} V 2 E_{n^{\prime}} L_{x} L_{z}}\right]^{1 / 2} \mathscr{M}_{f i},
$$

where

$$
\begin{aligned}
\mathscr{M}_{f i} & =\sqrt{ } 2 G_{\beta}\left[\bar{u}_{(p)}\left(\boldsymbol{P}^{\prime}\right) \gamma^{\mu}\left(g_{V}+g_{A} \gamma_{5}\right) u_{(n)}(\boldsymbol{P})\right] \\
& \times \int d y e^{i q_{y} y}\left[\bar{U}_{(e)}\left(y, n^{\prime}, \boldsymbol{p}_{y}^{\prime}\right) \gamma_{\mu} L u_{\left(\nu_{e}\right)}(\boldsymbol{k})\right]
\end{aligned}
$$

using the shorthand

$$
q_{y}=P_{y}+k_{y}-P_{y}^{\prime} .
$$

Note that Eq. (20) does not have a $\delta$-function corresponding to the conservation of $y$-component of momentum, because the latter is not a good quantum number in this problem. The transition rate can now be written as

$$
\left|S_{f i}\right|^{2} / T=\frac{1}{16}(2 \pi)^{3} \delta_{\xi^{\prime}}^{3}\left(P+k-P^{\prime}-p^{\prime}\right) \frac{E_{n^{\prime}}+m}{V^{3} \omega P_{0} P_{0}^{\prime} E_{n^{\prime}}}\left|\mathscr{M}_{f i}\right|^{2} .
$$

Putting in now the differential phase space for final particles, which is

$$
\frac{L_{x}}{2 \pi} d p_{x}^{\prime} \frac{L_{z}}{2 \pi} d p_{z}^{\prime} \frac{V}{(2 \pi)^{3}} d^{3} P^{\prime}
$$

where $V=L_{x} L_{y} L_{z}$, we can write the differential cross section as

$$
d \sigma=\frac{1}{64 \pi^{2}} \delta_{y}^{3}\left(P+k-P^{\prime}-p^{\prime}\right) \frac{E_{n^{\prime}}+m}{\omega P_{0} P_{0}^{\prime} E_{n^{\prime}}}\left|\mathscr{M}_{f i}\right|^{2} \frac{L_{x} L_{z}}{V} d p_{x}^{\prime} d p_{z}^{\prime} d^{3} P^{\prime} .
$$


The square of the matrix element, averaged over initial neutron spin, is given by

$$
\left|\mathscr{M}_{f i}\right|^{2}=G_{\beta}^{2} \ell_{\mu \nu} H^{\mu \nu} .
$$

Here, $H^{\mu \nu}$ is the hadronic part, which is

$$
\begin{aligned}
H^{\mu \nu} & =4\left(g_{V}^{2}+g_{A}^{2}\right)\left(P^{\mu} P^{\prime \nu}+P^{\nu} P^{\prime \mu}-g^{\mu \nu} P \cdot P^{\prime}\right) \\
& +4\left(g_{V}^{2}-g_{A}^{2}\right) m_{n} m_{p} g^{\mu \nu}+8 g_{V} g_{A} i \varepsilon^{\mu \nu \lambda \rho} P_{\lambda}^{\prime} P_{\rho} .
\end{aligned}
$$

The leptonic part $\ell_{\mu \nu}$ contains magnetic spinors:

$$
\ell_{\mu \nu}=\int d y \int d y_{\star} e^{i q_{y}\left(y-y_{\star}\right)} \operatorname{Tr}\left[P_{U}\left(y_{\star}, y, n^{\prime}, \boldsymbol{p}_{\star}^{\prime}\right) \gamma_{\mu} k \gamma_{\nu} L\right] .
$$

The integrations over $y$ and $y_{\star}$ can be exactly performed[4], yielding

$$
\ell_{\mu \nu}=\frac{2 \pi}{e B} \frac{1}{\left(E_{n^{\prime}}+m\right)}\left(\Lambda_{\mu} k_{\nu}+\Lambda_{\nu} k_{\mu}-k \cdot \Lambda g_{\mu \nu}-i \varepsilon_{\mu \nu \alpha \beta} \Lambda^{\alpha} k^{\beta}\right),
$$

where

$$
\begin{aligned}
\Lambda^{\alpha}= & {\left[I_{n}\left(\frac{q_{y}}{\sqrt{e B}}\right)\right]^{2}\left(p_{\|}^{\prime \alpha}-\widetilde{p}_{\|}^{\alpha}\right)+\left[I_{n-1}\left(\frac{q_{y}}{\sqrt{e B}}\right)\right]^{2}\left(p_{\|}^{\prime \alpha}+\widetilde{p}_{\|}^{\alpha}\right) } \\
& +2 M_{n} g_{2}^{\alpha} I_{n}\left(\frac{q_{y}}{\sqrt{e B}}\right) I_{n-1}\left(\frac{q_{y}}{\sqrt{e B}}\right) .
\end{aligned}
$$

We calculate the cross section in the rest frame of the neutron. The axes are chosen such that incoming neutrino is in the $x-z$ plane. Also, we assume that in the energy regime of interest, $\left|\boldsymbol{P}^{\prime}\right| \ll m_{p}$ so that the proton is non-relativistic. Then we obtain, for $n^{\prime} \neq 0$,

$$
\left|\mathscr{M}_{f i}\right|^{2}=\frac{16 \pi G_{\beta}^{2}}{e B} \frac{m_{n} m_{p}}{E_{n^{\prime}}+m}\left[\left(g_{V}^{2}+3 g_{A}^{2}\right) \omega \Lambda_{0}+\left(g_{V}^{2}-g_{A}^{2}\right) k_{z} \Lambda_{z}\right] .
$$

We now put it into Eq. (25). Integrations over $P_{x}^{\prime}$ and $P_{z}^{\prime}$ get rid of the $\delta$ functions. Integration over $p_{x}^{\prime}$ gives a factor $L_{y} e B$.

$$
d \sigma=\frac{G_{\beta}^{2}}{4 \pi} \frac{\delta\left(Q+\omega-E_{n^{\prime}}\right)}{\omega E_{n^{\prime}}}\left[\left(g_{V}^{2}+3 g_{A}^{2}\right) \omega \Lambda_{0}+\left(g_{V}^{2}-g_{A}^{2}\right) k_{z} \Lambda_{z}\right] d P_{y}^{\prime} d p_{z}^{\prime},
$$

where $Q=m_{n}-m_{p}$. Integration over $P_{y}^{\prime}$ can now be performed using the orthogonality of the Hermite polynomials. This ensures that the $M_{n}$-term of Eq. (30) vanishes. The other two give

$$
d \sigma_{n^{\prime}>0}=\frac{e B G_{\beta}^{2}}{2 \pi} \delta\left(Q+\omega-E_{n^{\prime}}\right)\left[\left(g_{V}^{2}+3 g_{A}^{2}\right)+\left(g_{V}^{2}-g_{A}^{2}\right) \frac{k_{z} p_{z}^{\prime}}{\omega E_{n^{\prime}}}\right] d p_{z}^{\prime} .
$$


The argument of the remaining $\delta$-function vanishes for two different values of $p_{z}^{\prime}$ which are equal and opposite. The term proportional to $g_{V}^{2}-g_{A}^{2}$ then cancels out and we obtain

$$
\sigma_{n^{\prime}>0}=\frac{e B G_{\beta}^{2}}{\pi}\left(g_{V}^{2}+3 g_{A}^{2}\right) \frac{Q+\omega}{\sqrt{(Q+\omega)^{2}-m^{2}-M_{n^{\prime}}^{2}}} .
$$

For $n^{\prime}=0$, however, even the second term of Eq. (30) vanishes, so we need to redo everything beginning from Eq. (31). The final result is:

$$
\sigma_{0}=\frac{e B G_{\beta}^{2}}{2 \pi}\left[\left(g_{V}^{2}+3 g_{A}^{2}\right)-\left(g_{V}^{2}-g_{A}^{2}\right) \frac{k_{z}}{\omega}\right] \frac{Q+\omega}{\sqrt{(Q+\omega)^{2}-m^{2}}} .
$$

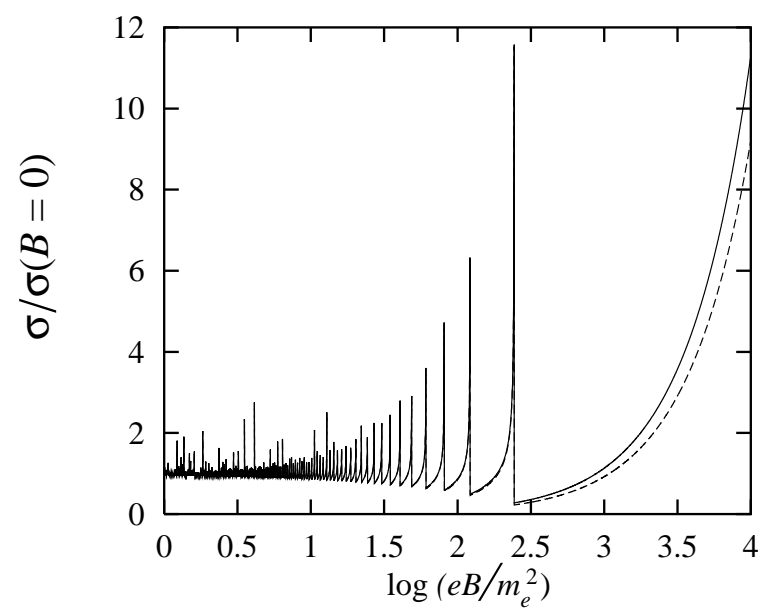

Figure 1: Total cross section as a function of the magnetic field, normalized to the cross section in the field-free case. The initial neutrino energy is $10 \mathrm{MeV}$. The solid and the dashed lines are for the initial neutrino momentum parallel and antiparallel to the magnetic field.

For any given energy of the initial states, there is a maximum possible value for $n^{\prime}$, dictated by Eq. (2). The total cross section is obtained by performing the sum over all possible $n^{\prime}$. We can then summarize our results as follows:

- $\sigma_{0}$ depends on the direction of neutrino momentum because of the term involving $k_{z}$.

- Total $\sigma$ always contains $\sigma_{0}$, and is hence asymmetric.

- The fractional amount of asymmetry depends on $n_{\max }^{\prime}$. It is maximum if $n_{\max }^{\prime}=0$, and decreases at smaller values of $B$ as more Landau levels contribute.

In Fig. 1, we show the cross section as a function of $B$ for neutrinos incident along and opposite to the direction of the magnetic field. For small values of 
$B$, the distinction between the two directions is not seen at the scale of the plot. However, when $B$ is so large that $n_{\max }^{\prime}=0$, the asymmetry is large. It is, in fact, as large as $18 \%$ between these two extreme directions. It seems highly plausible that such a large asymmetry in the cross section would produce enough momentum asymmetry to explain the pulsar kicks. These calculations were not finished at the time of the conference and therefore could not be presented[5].

There is however another important issue to be settled before the momentum asymmetry is calculated. Neutrinos undergo many other reactions inside a dense star. The inverse beta decay has the largest cross section for the $B=0$ case of course, but there is no guarantee that other cross sections are not larger in strong magnetic fields. Our preliminary calculations for the neutrino-electron elastic scattering show that it can increase by several orders of magnitude in a magnetic field. If that is the case, then the opacities have to be calculated taking these processes into account.

\section{References}

[1] J. C. D'Olivo, J. F. Nieves, P. B. Pal: Phys. Rev. D40 (1989) 3679; S. Esposito and G. Capone: Z. Phys. C70 (1996) 55; J. C. D'Olivo and J. F. Nieves: Phys. Lett. B383 (1996) 87; P. Elmfors, D. Grasso and G. Raffelt: Nucl. Phys. B479 (1996) 3.

[2] A. Kusenko and G. Segrè: Phys. Rev. Lett. 77 (1996) 4872.

[3] E. Roulet: JHEP 9801 (1998) 013.

[4] I. S. Gradshtein and I. M. Ryzhik: Table of integrals, series, and products (4th edition 1980, Academic Press). See result 1 in 7.376.

[5] Between the time of giving this talk and writing it up for the proceedings, we have finished the paper and submitted it for publication: K. Bhattacharya and P. B. Pal: hep-ph/9911498. This paper contains a lot of details of the entire calculation. 